\title{
Metric Perspectives of the Ricci Flow Applied to Disjoint Unions
}

Abstract: In this paper we consider compact, Riemannian manifolds $M_{1}, M_{2}$ each equipped with a oneparameter family of metrics $g_{1}(t), g_{2}(t)$ satisfying the Ricci flow equation. Adopting the characterization of super-solutions to the Ricci flow developed by McCann-Topping, we define a super Ricci flow for a family of distance metrics defined on the disjoint union $M_{1} \sqcup M_{2}$. In particular, we show such a super Ricci flow property holds provided the distance function between points in $M_{1}$ and $M_{2}$ is itself a super solution of the heat equation on $M_{1} \times M_{2}$. We also discuss possible applications and examples.

Keywords: super Ricci flow, disjoint union, heat kernel

MSC: 53C20, 53C23

DOI 10.2478/agms-2014-0011

Received March 3, 2014; accepted October 14, 2014

\section{Introduction}

For $i=1,2$, let $M_{i}$ be a compact $n$-dimensional Riemannian manifold equipped with a smooth family of metrics $g_{i}(t)$ satisfying Hamilton's Ricci flow equation [10]

$$
\frac{\partial g_{i}(t)}{\partial t}=-2 \operatorname{Ric}\left(g_{i}(t)\right)
$$

for $t \in\left[0, T_{i}\right)$. The short-time existence and uniqueness of solutions was demonstrated in [10] and we denote $T=\min \left(T_{1}, T_{2}\right)$. In this note, we consider the disjoint union $M_{1} \sqcup M_{2}$ equipped with a one-parameter family of distance metrics $D(t)$, for $t \in[0, T)$, so that $\left(M_{1} \sqcup M_{2}, D(t)\right)$ is a complete, compact metric space whose metric is compatible with the evolving metrics $g_{i}(t)$. That is to say, for each $t \in[0, T)$ and $i=1,2$

$$
\left.D(t)\right|_{M_{i}}=d_{g_{i}(t)},
$$

where $d_{g}$ denotes the distance metric induced by the Riemannian metric $g$. Following [14], we adopt the characterization of super Ricci flow solutions for an individual family of smooth Riemannian metrics to define a notion of super Ricci flow for the family of metric spaces $\left(M_{1} \sqcup M_{2}, D(t)\right)$; i.e.

Definition 1.1. With $M_{1}$ and $M_{2}$ as above, a family of distance metrics $D(t)$ on $M_{1} \sqcup M_{2}$, for $t \in[0, T)$, is called a super Ricciflow of the disjoint union $M_{1} \sqcup M_{2}$ provided whenever $0<a<b<T$ and $u(x, t): M_{1} \sqcup M_{2} \times(a, b) \rightarrow$ $\mathbb{R}$ is a solution to the heat equation on $M_{1} \sqcup M_{2}$, then

$$
\operatorname{Lip}(u, t):=\sup _{\substack{x \neq y \\ x, y \in M_{1} \sqcup M_{2}}} \frac{|u(x, t)-u(y, t)|}{D(t ; x, y)} \text { is non-increasing in } t .
$$

\footnotetext{
^Corresponding Author: Michael Munn: University of Missouri, Dept. of Mathematics, Columbia, MO 65201, USA, E-mail: munnm@missouri.edu

Sajjad Lakzian: Institutfür Angewandte Mathematik, Universität Bonn, Endenicher Allee 60, 53115 Bonn,Germany, E-mail: slakzian@gc.cuny.edu 
In Section 2, we recall work of von Renesse-Sturm [20] to clarify precisely what it means for $u(x, t)$ to be a solution to the heat equation on $M_{1} \sqcup M_{2}$ (see Definition 2.4 and the discussion therein).

We show

Theorem 1.2. For $i=1,2$, let $M_{i}$ be a compact, oriented $n$-dimensional manifold equipped with a smooth family of metrics $g_{i}(t)$ satisfying the Ricci flow equation (1.1) for $t \in\left[0, T_{i}\right)$ and let $T=\min \left(T_{1}, T_{2}\right)$. Consider the family of metric spaces $\left(M_{1} \sqcup M_{2}, D(t)\right)$ and the map

$$
\begin{aligned}
D: & {[0, T) \times\left(M_{1} \times M_{2}\right) \longrightarrow(0, \infty) } \\
& (t, x, y) \mapsto D(t ; x, y):=\text { distance between } x \in M_{1}, y \in M_{2}, \text { w.r.t. } D(t) .
\end{aligned}
$$

Letting $\Delta_{M_{1}^{t} \times M_{2}^{t}}$ denote the Laplacian on $\left(M_{1} \times M_{2}, g_{1}(t) \oplus g_{2}(t)\right)$, if

$$
\frac{\partial}{\partial t} D \geq \Delta_{M_{1}^{t} \times M_{2}^{t}} D,
$$

then the family of metrics $D(t)$ is a super Ricci flow of $M_{1} \sqcup M_{2}$.

Remark 1.3. The statement of Theorem 1.2 can be phrased slightly more generally in that $\left(M_{1}, g_{1}(t)\right)$ and $\left(M_{2}, g_{2}(t)\right)$ need only be super-solutions to the Ricci flow equation; i.e. $g_{i}(t)$ are super Ricci flows (see Definition 2.2) on $M_{i}, i=1,2$.

\subsection{Motivation}

To begin, we place the result of Theorem 1.2 in context by giving a motivation for considering such a family of metric spaces $\left(M_{1} \sqcup M_{2}, D(t)\right)$.

A primary advantage of Theorem 1.2 is that condition (1.4) is metric by nature and gives a sufficient condition for a family of distance metrics on the set $M_{1} \sqcup M_{2}$ to evolve in a way that is compatible with the smooth evolution of the Ricci flow for the Riemannian metrics on $M_{1}$ and $M_{2}$. This metric perspective allows for a more broad description of solutions to the Ricci flow which can persist through the development of singularities. See also recent work of Kleiner-Lott [12] which introduces the concept of singular Ricci flow to address the problem of flowing through singularities in the three-dimensional case.

The formation of singularities is an important phenomenon and intensely studied aspect of the Ricci flow and geometric evolution equations in general. We say a solution $(M, g(t))$ to the Ricci flow develops a Type I singularity at some finite time $T<\infty$, if

$$
\sup _{M \times[0, T)}(T-t)|\operatorname{Rm}(\cdot, r)|<+\infty
$$

The simplest example of a Type I singularity is the round sphere shrinking to a point in finite time and, in some sense, the formation of such singularities is a 'typical' property of the Ricci flow. Hamilton [10, 11] showed that the Ricci flow on a compact 3-manifold with positive Ricci curvature develops a Type I singularity and shrinks to a round point. Later, in [5], Böhm-Wilking show that a general compact $n$-dimensional manifold, for $n \geq 3$, with positive curvature operator also develops at Type I singularity in finite time.

Like the shrinking sphere, the results mentioned above describe global singularities of the Ricci flow; whereas, a local singularity occurs on a compact subset of the manifold while keeping the volume positive. Intuitively, a compact manifold shaped like a dumbbell develops a finite-time local singularity as the neck part of the dumbbell contracts. Rigorous examples of such local singularities were first produced for non-compact manifolds (initially by Simon [17] for warped product metrics on $\mathbb{R} x_{f} \mathbb{S}^{n}$ and then by Feldman-Ilmanen-Knopf on holomorphic line bundles over $\left.\mathbb{C P}^{n-1}\right)$. However, Angenent-Knopf $[1,2]$ were the first to exhibit rigorous examples of finite time Type I local singularities for compact manifolds. Specifically, they produce a class of rotationally symmetric metrics on $\mathbb{S}^{n+1}$, for $n \geq 2$, which develop neck-pinch singularities through the Ricci flow in finite-time. 
A solution $\left(M^{n+1}, g(t)\right)$ of the Ricci flow develops a neck-pinch singularity at time $T<\infty$ if there exists a time-dependent family of proper open subsets $N(t) \subset M^{n+1}$ and diffeomorphisms $\phi_{t}: \mathbb{R} \times \mathbb{S}^{n} \rightarrow N(t)$ such that $g(t)$ remains regular on $M^{n+1} \backslash N(t)$ and the pullback $\phi_{t}^{\star}\left(\left.g(t)\right|_{N(t)}\right)$ on $\mathbb{R} \times \mathbb{S}^{n}$ approaches the "shrinking cylinder" soliton metric

$$
d s^{2}+2(n-1)(T-t) g_{c a n}
$$

in $\mathcal{C}_{l o c}^{\infty}$ as $t \nearrow T$, where $g_{c a n}$ denotes the round metric on the unit sphere $\mathbb{S}^{n}$. In [1], the authors show how these neck pinch singularities arise for a class of metrics on $\mathbb{S}^{n+1}$. In [2], they derive detailed asymptotics of the profile of the solution near the singularity as well as comparable asymptotics for fully general neck pinches whose initial metric need need not be rotationally symmetric. Later, Angenent-Caputo-Knopf [3] extend this work by constructing smooth forward evolutions of the Ricci flow starting from initial singular metrics which arise from rotationally symmetric neck pinches on $\mathbb{S}^{n+1}$ by passing to the limit of a sequence of Ricci flows with surgery.

Together [1-3] provide a framework for developing the notion of a 'canonically defined Ricci flow through singularities' as conjectured by Perelman in [15]. Up to this point, continuing a solution of the Ricci flow past a singular time $T<\infty$ required surgery and a series of carefully made choices so that certain crucial estimates remain bounded through the flow. A complete 'canonical Ricci flow through singularities' would avoid these arbitrary choices and would be broad enough to address all types of singularities that arise in the Ricci flow.

The motivation for the current paper follows from this work of Angenent-Knopf and Angenent-CaputoKnopf, though our result allows for application in a more general context. Since the smooth forward evolution described in [3] performs a topological surgery on $\mathbb{S}^{n+1}$ at the singular time $T$, all future times will consist of two disjoint smooth Ricci flows on a pair of manifolds. Furthermore, although the metric $g(t)$ is no longer a smooth Riemannian metric at the singular time $t=T$, the space $\mathbb{S}^{n+1}$ does retain the structure of a metric space with distance metric denoted $d_{T}$ arising from the convergence of the distance metrics $d_{t}$ on $\left(\mathbb{S}^{n+1}, g(t)\right)$ through the evolution. Furthermore, these spaces converge as metric spaces in the Gromov-Hausdorff sense; i.e.

$$
\lim _{t \nearrow T} d_{G H}\left(\left(\mathbb{S}^{n+1}, d_{t}\right),\left(\mathbb{S}^{n+1}, d_{T}\right)\right)=0 .
$$

Our Theorem 1.2 gives a metric context in which to understand the evolution of the Ricci flow for $t>T$, after this singularity develops, by treating the disjoint union of two separate Ricci flows as a single metric space with distance metrics evolving in a way compatible to the flow.

The remainder of this paper is organized as follows. In Section 2, we recall the characterization of the super Ricci flow given by McCann-Topping for compact Riemannian manifolds which motivates our Definition 1.1. Also, we recall a construction of von Renesse-Sturm [20] and use a generalization of the Trotter-Chernov product formula for time dependent operators to describe solutions to the heat equation on the disjoint union $M_{1} \sqcup M_{2}$. With these definitions and context in place, we then prove Theorem $1.2 \mathrm{in} \mathrm{Section} 3$ and give implications. In Section 4, we give some simple examples of metric constructions for the super Ricci flow for disjoint unions of two smooth Riemannian manifolds. In particular, we consider the situation when $M_{1} \cong M_{2}$ and consider the case of the flat torus and the round sphere.

\section{Background}

As we hope to make clear, our current results tie together a progression of ideas which originated with a 2005 paper by M. von Renesse and K.T. Sturm [20], although its true origins can be recognized in earlier work of Bakry-Emery [4], Cordero-Erausquin, McCann, Schmuckenschläger [6, 7] and others. 


\subsection{Metric characterizations of Ricci curvature lower bounds and the Ricci flow}

In [20], von Renesse-Sturm characterize uniform lower Ricci curvature bounds of smooth Riemannian manifolds $\left(M^{n}, g\right)$ using various convexity properties of the entropy as well as transportation inequalities of volume measures, heat kernels, and gradient estimates of the heat semigroup on $M^{n}$. In fact, the metric nature of the ideas presented in that paper introduced into the literature a discussion of so called "synthetic" definitions of Ricci curvature lower bounds which do not rely on the underlying smooth structure of the manifold and thus lend themselves to spaces lacking that smooth structure, such as metric measure spaces, Alexandrov spaces, or general metric spaces.

We state here only a small part the results in [20] which are relevant to our later discussion. First a bit of notation: Let $\left(M^{n}, g\right)$ be a smooth, connected, complete Riemannian manifold of dimension $n$. Denoting the heat kernel on $M^{n}$ by $p_{s}(x, y)$ one can define the operators $p_{s}: C_{c}^{\infty}(M) \rightarrow C^{\infty}(M)$ and $p_{s}: L^{2}(M) \rightarrow L^{2}(M)$ by $f \mapsto p_{s} f(x):=\int_{M} p_{s}(x, y) f(y) d \operatorname{Vol}(y)$. They prove

Theorem 2.1. (c.f. [20]). For any smooth, complete Riemannian manifold $\left(M^{n}, \mathrm{~g}\right), \mathrm{Ric}_{M} \geq 0$ if and only if for all bounded $f \in \mathcal{C}^{\text {Lip }}(M)$ and all $s>0$,

$$
\operatorname{Lip}\left(p_{s} f\right) \leq \operatorname{Lip}(f)
$$

Later McCann-Topping [14] took a dynamic approach and reinterpreted the work of von Renesse-Sturm in relation to a family of metrics $g(t)$ on $M$ evolving by (1.1). Specifically, they characterize super solutions of the Ricci flow by the contractivity of mass diffusions backwards in time. We refer to a super solutions of the Ricci flow as a super Ricci flow. That is

Definition 2.2. For a compact, oriented $n$-dimensional manifold, a super Ricci flow is a smooth family $g(t)$ of metrics on $M, t \in[0, T]$, such that at each $t \in(0, T)$ and each point on $M$, one has

$$
\frac{\partial g}{\partial t}+2 \operatorname{Ric}(g(t)) \geq 0 \text {. }
$$

In addition, and more closely related to our purposes, they prove the following

Theorem 2.3. (c.f. [14]). Let $M^{n}$ be a compact, Riemannian manifold of dimension $n$. A smooth one-parameter family of metrics for $t \in[0, T)$ is a super Ricci flow if and only if whenever $0<a<b<T$ and $f: M \times(a, b) \rightarrow \mathbb{R}$ is a solution to $\frac{\partial f}{\partial t}=\Delta_{g(t)} f$, then

$$
\operatorname{Lip}(f, t):=\sup _{x \neq y} \frac{|f(x, t)-f(y, t)|}{d(x, y, t)} \text { is non-increasing in } t .
$$

The quantity $\operatorname{Lip}(f, t)$ is the Lipschitz constant of $f(\cdot, t)$ evaluated using the metric $g(t)$. It is precisely this characterization which we use to define the notion of a super Ricci flow for the disjoint union of two evolving Riemannian manifolds. However, we must first make sense of the local representation for the heat kernel on $M_{1} \sqcup M_{2}$ in order to describe what it means for a function $u(x, t)$ on $M_{1} \sqcup M_{2}$ to solve the heat equation.

\subsection{Deriving heat kernel operators via the metric and measure}

In [20], von Renesse-Sturm study smooth, connected complete $n$-dimensional Riemannian manifolds and characterize a uniform lower Ricci curvature bounds using, among other things, heat kernels and transportation inequalities for uniform distribution measures on distance spheres. One striking advantage of these characterizations is that they depend only on the metric and measure of the underlying smooth Riemannian manifold and thus allow for a notion of a Ricci curvature lower bound depending solely this basic, non-smooth data. In fact, these characterizations ultimately led to the current definitions of Ricci curvature for arbitrary metric measure spaces introduced independently by Lott-Villani and Sturm [13, 18, 19]. We recall now a part of these original results of von Renesse-Sturm. 
Following the comment at the end of Section 1 of [20], one can view a smooth, connected Riemannian manifold $(M, g)$ as a separable metric measure space $\left(M, d_{g}, \mathrm{Vol}_{g}\right)$ and define a family of Markov operators $\sigma_{r}$ acting on the set of bounded Borel measurable functions by $\sigma_{r} f(x)=\int_{M} f(y) d \sigma_{r, x}(y)$, where the measure $\sigma_{r, x}$ is defined as

$$
\sigma_{r, x}(A):=\frac{\operatorname{Vol}_{g}(A \cap \partial B(x, r))}{\operatorname{Vol}_{g}(\partial B(x, r))}, \quad A \in \mathcal{B}(M) .
$$

Here $B(x, r)$ denotes the ball of radius $r$ centered at $x$. By the Arzela-Ascoli theorem and applying the TrotterChernov product formula [8], there exists a subsequence such that for all $s \geq 0$ and all bounded $f \in C^{\operatorname{Lip}}(M)$, it follows that $\lim _{j \rightarrow \infty}\left(\sigma_{\sqrt{2 n s / j}}\right)^{j} f(x)$ exists and converges uniformly in $x \in M$ and locally uniformly in $s \geq 0$. In fact, letting $p_{s}(x, y)$ denote the minimal smooth heat kernel on $M^{n}$ (i.e. the positive fundamental solution to $\left.\left(\Delta-\frac{\partial}{\partial s}\right) p_{s}(x, y)=0\right)$ then it follows that

$$
p_{s} f(x)=\lim _{j \rightarrow \infty}\left(\sigma_{\sqrt{2 n s / j}}\right)^{j} f(x) .
$$

Thus, utilizing (2.3) we describe solutions to the heat equation for an arbitrary metric measure space $(M, d, m)$ independently of the smooth structure.

\subsection{Constructing a heat kernel on $M_{1} \sqcup M_{2}$}

Consider a single smooth manifold evolving by the Ricci flow. That is, let $g(t)$ be a family of metrics on $M^{n}$ satisfying (1.1) for $t \in[0, T), T>0$. At each time $t$, just as in (2.2), we define the normalized Riemannian uniform distribution on spheres centered at $x \in(M, g(t))$ of radius $r>0$ by

$$
\sigma_{r, x}^{t}(A):=\frac{\mathcal{H}^{n-1}\left(A \cap \partial B^{t}(x, r)\right)}{\mathcal{H}^{n-1}\left(\partial B^{t}(x, r)\right)}, \quad A \in \mathcal{B}(M),
$$

where $B^{t}(x, r)$ denotes the ball of radius $r$ centered at $x$ with respect to the fixed metric $g(t)$. As before, we have a family of Markov operators $\sigma_{r}^{t}$ on the set of bounded Borel-measurable functions $(M, g(t))$ defined above replacing $\sigma_{r}$ by $\sigma_{r}^{t}$ and integrating over $(M, g(t))$. It follows that (for a subsequence) as $j \rightarrow \infty$,

$$
\left(\sigma_{\sqrt{2 n s / j}}^{t}\right)^{j} f(x) \rightarrow p_{s}^{t} f(x)=e^{s \Delta_{g(t)}} f(x)
$$

uniformly in $x \in(M, g(t))$ and locally uniformly in $s \geq 0$, for all bounded $f \in \mathcal{C}^{\operatorname{Lip}}\left(M^{n}, g(t)\right)$.

Consider now $M \times[0, T)$, the entire space-time where the Ricci flow is defined. Let $B$ denote the Banach space $\mathcal{C}^{\mathrm{Lip}}\left(M^{n}, g(t)\right)$ with the sup-norm and $\mathcal{L}(B)$ the space of bounded linear operators on $B$. For each $t$, define functions $F_{t}:[0, \infty) \rightarrow \mathcal{L}(\mathcal{B})$ where

$$
F_{t}(s)=e^{s \Delta_{g(t)}}
$$

Note that $F_{t}(0)=I d$ for every $t \in[0, T)$ and, for any $f \in B$,

$$
F_{t}^{\prime}(0) f=\lim _{s \downarrow 0} \frac{F_{t}(s) f-f}{s}=\lim _{s \downarrow 0} \frac{e^{s \Delta_{g(t)}} f-f}{s}=\Delta_{g(t)} f .
$$

Thus, it follows from the generalized Trotter-Chernov product formula (see [22], Main Theorem) applied to the time-dependent operators given by (2.6), that for any function $u: M \times(0, T) \rightarrow \mathbb{R}$ solving the initial value problem

$$
\left\{\begin{array}{l}
\frac{d}{d t} u(\mathbf{x}, t)=\Delta_{g(t)} u(\mathbf{x}, t) \\
u(\mathbf{x}, 0)=f(\mathbf{x})
\end{array}\right.
$$

there exists a corresponding one-parameter family of bounded linear operators $U(t, 0)_{0 \leq t \leq T}$ in $B$ such that $u(\mathbf{x}, t)=U(t, 0) f(\mathbf{x})$. Therefore, for all $0 \leq t \leq T$ we have

$$
U(t, 0)=\lim _{m \rightarrow \infty} \prod_{i=m-1}^{0} F_{\frac{i}{m} t}\left(\frac{t}{m}\right)=\lim _{m \rightarrow \infty} \prod_{i=m-1}^{0} e^{\frac{t}{m} \Delta_{g\left(\frac{i}{m} t\right)}}
$$


with convergence of the limit in the strong operator topology of $\mathcal{L}(B)$. Combining this (2.5) we can further write, for any $f \in B$,

$$
u(\mathbf{x}, t)=U(t, 0) f(\mathbf{x})=\lim _{m \rightarrow \infty} \prod_{i=m-1}^{0} \lim _{j \rightarrow \infty}\left(\begin{array}{c}
\sigma^{\frac{i}{m} t} \\
\sqrt{\frac{2 n t}{j m}}
\end{array}\right)^{j} f(\mathbf{x}) .
$$

As noted earlier, this description gives a metric measure characterization of solutions to the heat equation on the evolving manifold $(M, g(t))$.

Finally, we turn our attention to the situation of the current paper and use the characterization above to describe solutions for the heat equation on $M_{1} \sqcup M_{2}$. Note that the description in (2.9) is locally defined and thus allows for generalization to the disjoint union $M_{1} \sqcup M_{2}$. Indeed, as $j \rightarrow \infty$ the operators $\sigma^{\frac{i}{m} t} \sqrt{\frac{2 n t}{\bar{j} m}}$ are ultimately restricted to individual components $M_{1}$ or $M_{2}$ of $M_{1} \sqcup M_{2}$ depending on whether $x \in M_{1}$ or $x \in M_{2}$ (resp.). Motivated by the discussion above we define

Definition 2.4. Let $\left(M_{i}, g_{i}(t)\right)$, for $i=1,2$, be compact Riemannian manifolds supporting smooth families of metrics satisfying the Ricci flow equation given by (1.1) for $t \in\left[0, T_{i}\right)$. Also, let $D(t)$ be a family of distance functions on $M_{1} \sqcup M_{2}$ so that each $t \in\left[0, \min \left(T_{1}, T_{2}\right)\right)$ we have $\left(M_{1} \sqcup M_{2}, D(t)\right)$ is a complete, compact metric space compatible with the family of metrics $g_{i}(t)$ on $M_{i}$ resp.; i.e. for $i=1$, 2 ,

$$
\left.D(t)\right|_{M_{i}}=d_{g_{i}(t)},
$$

and such that

$$
\frac{\partial}{\partial t} D(t ; x, y) \geq \Delta_{M_{1}^{t} \times M_{2}^{t}} D(t ; x, y), \quad \text { for } x \in M_{1}, y \in M_{2} .
$$

A function $u: M_{1} \sqcup M_{2} \times(0, T) \rightarrow \mathbb{R}$ is said to solve the initial value problem (2.7) on $M_{1} \sqcup M_{2}$ for $f \in$ $\mathrm{e}^{\text {Lip }}\left(M_{1} \sqcup M_{2}, D(t)\right)$, provided

$$
u(\mathbf{x}, t)=\lim _{m \rightarrow \infty} \prod_{i=m-1}^{0} \lim _{j \rightarrow \infty}\left(\begin{array}{c}
\sigma^{\frac{i}{m} t} \\
\sqrt{\frac{2 n t}{j m}}
\end{array}\right)^{j} f(\mathbf{x}) .
$$

Note that

Lemma 2.5. Let $\left(M_{i}, g_{i}(t)\right)$, for $i=1,2$, and $\left(M_{1} \sqcup M_{2}, D(t)\right)$ be as above and suppose $D(0 ; x, y)>0$ for all $x \in M_{1}, y \in M_{2}$. A function $u: M_{1} \sqcup M_{2} \times(0, T) \rightarrow \mathbb{R}$ solves the initial value problem (2.7) on $M_{1} \sqcup M_{2}$ if and only if $\left.u\right|_{M_{i}}$ and satisfies smooth heat equation on $M_{i}$, for $i=1,2$.

Proof. First, note that if $D(0 ; x, y)>0$ for $x \in M_{1}, y \in M_{2}$ at the initial time $t=0$, then by the maximum principle (see, for example, Theorem 3.1.1 of [21]) we have

$$
D(t ; x, y)>0 \text {, for all } t>0 \text { and } x \in M_{1}, y \in M_{2} .
$$

For a fixed $t$, it follows that the measures $\sigma_{r, x}^{t}$ when defined on $M_{1} \sqcup M_{2}$ agree with $\left.\sigma_{r}^{t}\right|_{M_{i}}$ for $x \in M_{i}$ provided $r$ is taken small enough; namely $r<\inf _{x \in M_{1}, y \in M_{2}} D(t ; x, y)$. Thus, for $j$ large enough it follows that

$$
\sigma^{\frac{i}{m} t}=\left.\sigma^{\frac{i}{m} t}\left|\sqrt{\frac{2 n t}{j m}}\right|\right|_{M_{i}} .
$$

Now for $u: M_{1} \sqcup M_{2} \times(0, T) \rightarrow \mathbb{R}$ which satisfies the IVP given in (2.7) we have that

$$
\left.u(\mathbf{x}, t)\right|_{M_{1}}=\lim _{m \rightarrow \infty} \prod_{i=m-1}^{0} \lim _{j \rightarrow \infty}\left(\begin{array}{c}
\sigma^{\frac{i}{m} t} \\
\sqrt{\frac{2 n t}{j m}}
\end{array} \mid\right)_{M_{1}}^{j} f(\mathbf{x}) .
$$

As pointed out in the discussion above, for a smooth Riemannian manifold $M_{1}$ whose heat kernel is denoted by $p_{s}(x, y)$, we have

$$
p_{s} f(x)=\lim _{j \rightarrow \infty}\left(\sigma_{\sqrt{2 n s / j}}\right)^{j} f(x) .
$$


Thus, we can write using the notation as before where $F_{t}(s)=e^{s \Delta_{g(t)}}=p_{s}^{t}$,

$$
\left.u(\mathbf{x}, t)\right|_{M_{i}}=\lim _{m \rightarrow \infty} \prod_{i=m-1}^{0} p_{\frac{t}{m}}^{\frac{i}{m} t} f(\mathbf{x})=\lim _{m \rightarrow \infty} \prod_{i=m-1}^{0} F_{\frac{i}{m} t}\left(\frac{t}{m}\right) f(\mathbf{x})=U(t, 0) f(\mathbf{x}) .
$$

Thus, by the generalized Trotter product formula and (2.8), it follows that $\left.u(\mathbf{x}, t)\right|_{M_{1}}$ solves the heat equation on $M_{1}$. In precisely the same way, we verify that $\left.u\right|_{M_{2}}$ also satisfies the heat equation on $M_{2}$.

Furthermore, suppose some function $u(\mathbf{x}, t)$ defined on $M_{1} \sqcup M_{2}$ when restricted to either $M_{i}$ satisfies the heat equation on that component. Again by (2.13) it follows that $u(\mathbf{x}, t)$ satisfies the IVP on the disjoint union $M_{1} \sqcup M_{2}$.

\section{Proof of Theorem 1.2 and consequences}

Proof of Theorem 1.2. With $M_{i}$ as above, let $u_{i}: M_{i} \times(0, T)$ be solutions to $\frac{\partial u_{i}}{\partial t}=\Delta_{g_{i}(t)} u_{i}, i=1,2$. Consider the disjoint union $M_{1} \sqcup M_{2}$ and define a function $u: M_{1} \sqcup M_{2} \times(0, T) \rightarrow \mathbb{R}$ by

$$
u(x, t)= \begin{cases}u_{1}(x, t), & \text { when } x \in M_{1} \\ u_{2}(x, t), & \text { when } x \in M_{2}\end{cases}
$$

Recall, by assumption

$$
D\left(t ; m_{1}, m_{2}\right)>0 \text {, for all } m_{1} \in M_{1}, m_{2} \in M_{2}, t>0 \text {, }
$$

so by Lemma 2.5, the function $u(x, t)$ satisfies the heat equation on $M_{1} \sqcup M_{2}$. Note that for any $t \in[0, T)$, there exists $p, q \in\left(M_{1} \sqcup M_{2}, D(t)\right)$ such that

$$
\operatorname{Lip}(u, t)=\frac{|u(p, t)-u(q, t)|}{D(t ; p, q)} .
$$

By Theorem 2.3 in [14], for fixed $p, q \in M_{i}$, the property that $\operatorname{Lip}(u, t)$ is non-increasing as a function of $t$ is equivalent to $g_{i}(t)$ being a solution to the super Ricci flow. Thus, we are done since each $\left(M_{i}, g_{i}(t)\right)$ in fact solves (1.1) by assumption and so obviously (2.1). Therefore, we focus on the case when the Lipschitz constant of $u$ is achieved by a point in $M_{1}$ and a point in $M_{2}$.

Fix $t \in(0, T)$. Without loss of generality, assume the value of $\operatorname{Lip}(u, t)$ is attained by the points $p \in$ $M_{1}, q \in M_{2}$. In a neighborhood sufficiently near $(p, q) \in M_{1} \times M_{2}$, we may also assume (without loss of generality) that $u_{1}(x, t)-u_{2}(y, t) \geq 0$ so that the function on $M_{1} \times M_{2}$ given by

$$
(x, y) \mapsto \frac{u_{1}(x, t)-u_{2}(y, t)}{D(t ; x, y)}
$$

is nonnegative and has an absolute maximum at the point $(p, q)$. Therefore,

$$
\left.\nabla\left(\frac{u_{1}(x, t)-u_{2}(y, t)}{D(t ; x, y)}\right)\right|_{(p, q)}=0,
$$

and

$$
\left.\Delta\left(\frac{u_{1}(x, t)-u_{2}(y, t)}{D(t ; x, y)}\right)\right|_{(p, q)} \leq 0 .
$$

Furthermore, for points $x, y \in M_{1} \sqcup M_{2}$ sufficiently close to $p \in M_{1}$ and $q \in M_{2}$ (resp.) it follows from (3.2) that $u_{1}(x, t)-u_{2}(y, t)=\left.u\right|_{M_{1}}(x, t)-\left.u\right|_{M_{2}}(y, t)=u(x, t)-u(y, t)$.

To simplify notation, set $\bar{u}(x, y, t)=u_{1}(x, t)-u_{2}(y, t)$. From (3.4) we have

$$
\nabla\left(\frac{\bar{u}}{D(t)}\right)=\frac{D(t) \nabla \bar{u}-\bar{u} \nabla D(t)}{(D(t))^{2}}=0,
$$


and thus

$$
\bar{u} \nabla D(t)=D(t) \nabla \bar{u}
$$

To evaluate (3.5), note that

$$
\begin{aligned}
\nabla^{2}\left(\frac{\bar{u}}{D(t)}\right) & =\frac{(D(t))^{2}\left(\nabla D(t) \nabla \bar{u}+D(t) \nabla^{2} \bar{u}-\nabla \bar{u} \nabla D(t)-\bar{u} \nabla^{2} D(t)\right)-2 D(t) \nabla D(t)(D(t) \nabla \bar{u}-\bar{u} \nabla D(t))}{(D(t))^{4}} \\
& =\frac{\nabla^{2} \bar{u}}{D(t)}-\bar{u} \frac{\nabla^{2} D(t)}{(D(t))^{2}}-\frac{\nabla D(t) \otimes \nabla \bar{u}}{(D(t))^{2}}-\frac{\nabla \bar{u} \otimes \nabla D(t)}{(D(t))^{2}}+2 \frac{\bar{u} \nabla D(t) \otimes \nabla D(t)}{(D(t))^{3}} ;
\end{aligned}
$$

and, therefore

$$
\begin{aligned}
\Delta\left(\frac{\bar{u}}{D(t)}\right) & =\operatorname{tr} \nabla^{2}\left(\frac{\bar{u}}{D(t)}\right) \\
& =\operatorname{tr} \frac{(D(t))^{2}\left(\nabla D(t) \nabla \bar{u}+D(t) \nabla^{2} \bar{u}-\nabla \bar{u} \nabla D(t)-\bar{u} \nabla^{2} D(t)\right)-2 D(t) \nabla D(t)(D(t) \nabla \bar{u}-\bar{u} \nabla D(t))}{(D(t))^{4}} \\
& =\frac{\Delta \bar{u}}{D(t)}-\frac{\bar{u} \Delta D(t)}{(D(t))^{2}}-2 \frac{\langle\nabla D(t), \nabla \bar{u}\rangle}{(D(t))^{2}}+2 \frac{\bar{u}|\nabla D(t)|^{2}}{(D(t))^{3}},
\end{aligned}
$$

where we used (3.7) to evaluate in the last term. Furthermore, using (3.7) to write $\nabla \bar{u}=\frac{\bar{u} \nabla D(t)}{D(t)}$, we have

$$
2 \frac{\langle\nabla D(t), \nabla \bar{u}\rangle}{(D(t))^{2}}=\frac{\left\langle\nabla D(t), \frac{\bar{u} \nabla D(t)}{D(t)}\right\rangle}{(D(t))^{2}}=2 \frac{\bar{u}|\nabla D(t)|^{2}}{(D(t))^{3}}
$$

which implies

$$
\left.\Delta\left(\frac{\bar{u}}{D(t)}\right)\right|_{(p, q)}=\frac{\Delta \bar{u}}{D(t)}-\frac{\bar{u} \Delta D(t)}{(D(t))^{2}}
$$

So, by (3.5), it follows that at $(p, q)$

$$
\frac{\Delta \bar{u}}{D(t)} \leq \frac{\bar{u} \Delta D(t)}{(D(t))^{2}}
$$

or, equivalently,

$$
\Delta \bar{u}(p, q) \leq \frac{\bar{u}(p, q)}{D(t ; p, q)} \Delta D(t ; p, q) .
$$

By assumption, $\frac{\partial}{\partial t} D(t) \geq \Delta D(t)$, and since $\frac{\bar{u}}{D(t)} \geq 0$ we get

$$
\Delta \bar{u} \leq \frac{\bar{u}}{D(t)} \frac{\partial D(t)}{\partial t},
$$

and, thus, since $\bar{u}:\left(M_{1} \times M_{2}\right) \times(0, T) \rightarrow \mathbb{R}$ solves the heat equation by Lemma 2.5,

$$
\frac{\partial \bar{u}}{\partial t} \leq \frac{\bar{u}}{D(t)} \frac{\partial D(t)}{\partial t} .
$$

Finally, note that

$$
\frac{\partial}{\partial t} \operatorname{Lip}(u, t)=\frac{\partial}{\partial t} \sup _{\substack{x \neq y \\ x \in M_{1}, y \in M_{2}}} \frac{|u(x, t)-u(y, t)|}{D(t ; x, y)}=\sup _{\substack{x \neq y \\ x \in M_{1}, y \in M_{2}}} \frac{D(t) \frac{\partial \bar{u}}{\partial t}-\bar{u} \frac{\partial D(t)}{\partial t}}{(D(t ; x, y))^{2}}
$$

Since (3.13) holds for any pair of points which achieve the Lipschitz constant, it follows that $\frac{\partial}{\partial t} \operatorname{Lip}(u, t) \leq$ 0 and thus we have $\operatorname{Lip}(u, t)$ is decreasing as a function of $t$ and we are done.

This can be easily generalized to address additional components. 
Corollary 3.1. For $i=1,2, \cdots, k$, let $\left(M_{i}, g_{i}(t)\right)$ be compact $n$-dimensional manifolds whose metrics $g_{i}(t)$ satisfy (1.1) for $t \in\left[0, T_{i}\right)$. Consider a family of metric spaces $\left(M_{1} \sqcup M_{2} \sqcup \cdots M_{k}, D(t)\right)$ for $t \in(0, T), T=$ $\min \left(T_{1}, T_{2}, \cdots, T_{k}\right)$ and suppose that $D(t)$ satisfies (1.4) for all $x \in M_{i}, y \in M_{j}$ with $i \neq j$, then the family of metrics $D(t)$ is a super Ricci flow of $M_{1} \sqcup M_{2} \sqcup \cdots \sqcup M_{k}$.

Furthermore, considering $\left(M_{1} \sqcup M_{2}, D(t)\right)$ as a family of metric spaces, the evolution inequality given in (1.4) also provides control on how the distance between $M_{1}$ and $M_{2}$ changes over time. Namely, if at the initial time $t=0$ we have $D(0 ; x, y) \geq c>0$, then by a direct application of the maximum principle, $D(t ; x, y) \geq c$ for all $t>0$.

Corollary 3.2. Given $M_{i}$ as above and a family of metric spaces formed from the disjoint union $\left(M_{1} \sqcup M_{2}, D(t)\right)$ such that (1.4) holds. If at the initial time $D(0 ; x, y) \geq c>0$ for some $c \in \mathbb{R}$, then $D(t ; x, y) \geq c$, for all $t>0$.

Remark 3.3. Note that while the condition $\frac{\partial}{\partial t} D \geq \Delta_{M_{1}^{t} \times M_{2}^{t}} D$ alone isn't enough to guarantee that a given distance metric $D$ at time $t=0$ between $M_{1}$ and $M_{2}$ will remain a distance metric for all $t>0$, we restrict our attention to only those families $D(t)$ which, in fact, are distance metrics. In Section 4 we give simple constructions which verify that the class of such distance functions on $M_{1} \sqcup M_{2}$ is nonempty.

\section{Examples}

To better illustrate the content of Theorem 1.2 we mention a few simple examples. In general, for $\left(M_{1}, g_{1}(t)\right)$ and $\left(M_{2}, g_{2}(t)\right)$ as in Section 1, a family of distance metrics $D(t)$ on $M_{1} \sqcup M_{2}$ for $t \in[0, T)$ is a family of non-negative functions

$$
D(t): M_{1} \sqcup M_{2} \times M_{1} \sqcup M_{2} \rightarrow \mathbb{R}
$$

such that the following properties hold. For $\mathbf{a}, \mathbf{b}, \mathbf{c} \in M_{1} \sqcup M_{2}$, and all $t \in[0, T)$,

- $D(t)(\mathbf{a}, \mathbf{b})=0$ if and only if $\mathbf{a}=\mathbf{b}$

- $D(t)(\mathbf{a}, \mathbf{b})=D(t)(\mathbf{b}, \mathbf{a})$

- $D(t)(\mathbf{a}, \mathbf{b}) \leq D(t)(\mathbf{a}, \mathbf{c})+D(t)(\mathbf{c}, \mathbf{b})$

Thus, we require these properties to hold implicitly in the statement of Theorem 1.2. Note, however, that the metric $D(t)$ is not an intrinsic distance as $M_{1} \sqcup M_{2}$ is disconnected.

Consider the case where $\left(M_{1}, g_{1}(0)\right) \cong\left(M_{2}, g_{2}(0)\right)$ and thus $g_{1}(t)=g_{2}(t)$ for all $t$ satisfying (1.1) by uniqueness. Set $D(t)$ on $M_{1} \sqcup M_{2}$ to be

$$
D(t ; \mathbf{a}, \mathbf{b})= \begin{cases}d_{g_{i}(t)}(\mathbf{a}, \mathbf{b}), & \text { if } \mathbf{a}, \mathbf{b} \in M_{i} \\ \sqrt{L^{2}(t)+d_{g_{i}(t)}^{2}(\phi(\mathbf{a}), \mathbf{b})}, & \text { if } \mathbf{a} \in M_{1}, \mathbf{b} \in M_{2} \text { or } \mathbf{a} \in M_{2}, \mathbf{b} \in M_{1},\end{cases}
$$

where $\phi: M_{1} \rightarrow M_{2}$ is the identity map and $L(t)$ depends only on $t$. Note that each of the properties for $D(t)$ to be a distance function hold naturally in this construction.

Now letting $d_{t}$ denote $d_{g_{i}(t)}$ where there is no confusion since $g_{1}(t)=g_{2}(t)$ and considering $d_{t}$ and $D(t)$ as maps on $M_{1} \times M_{2}$, we can relate $\Delta_{M_{1}^{t} \times M_{2}^{t}} D(t)$ to $\Delta_{M_{1}^{t} \times M_{2}^{t}} d_{t}$. Computing in local coordinates we have

$$
\begin{aligned}
\Delta(D(t))^{2} & =\frac{1}{\sqrt{|g|}} \partial_{i}\left(\sqrt{|g|} g^{i j} \partial_{j}(D(t))^{2}\right) \\
& =\frac{1}{\sqrt{|g|}} \partial_{i}\left(\sqrt{|g|} g^{i j} 2 D(t) \partial_{j} D(t)\right) \\
& =2 g^{i j} \partial_{i} D(t) \partial_{j} D(t)+2 D(t) \Delta D(t)
\end{aligned}
$$


and, directly we find $\Delta(D(t))^{2}=\Delta\left(L^{2}(t)+d_{t}^{2}\right)=\Delta d_{t}^{2}=2\left|\nabla d_{t}\right|^{2}+2 d_{t} \Delta d_{t}$, since in this simple example we assume that $L(t)$ depends only on $t$. Thus,

$$
\begin{aligned}
d_{t} \Delta_{M_{1}^{t} \times M_{2}^{t}} d_{t} & =\frac{1}{2} \Delta_{M_{1}^{t} \times M_{2}^{t}}(D(t))^{2}-\left|\nabla^{M_{1}^{t} \times M_{2}^{t}} d_{t}\right|_{g(t)}^{2} \\
& =g_{t}^{i j} \partial_{i} D(t) \partial_{j} D(t)+D(t) \Delta_{M_{1}^{t} \times M_{2}^{t}} D(t)-\left|\nabla^{M_{1}^{t} \times M_{2}^{t}} d_{t}\right|_{g(t)}^{2}
\end{aligned}
$$

Therefore,

$$
\Delta_{M_{1}^{t} \times M_{2}^{t}} D(t)=\frac{d_{t}}{D(t)} \Delta_{M_{1}^{t} \times M_{2}^{t}} d_{t}+\frac{\left|\nabla^{M_{1}^{t} \times M_{2}^{t}} d_{t}\right|_{g(t)}^{2}}{D(t)}-\frac{1}{D(t)} g_{t}^{i j} \partial_{i} D(t) \partial_{j} D(t)
$$

Noting that $\partial_{i} D(t)=\frac{d_{t}}{D(t)} \partial_{i} d_{t}$ thus, we can further simplify the last term in the expression above to give

$$
\Delta_{M_{1}^{t} \times M_{2}^{t}} D(t)=\frac{d_{t}}{D(t)} \Delta_{M_{1}^{t} \times M_{2}^{t}} d_{t}+\frac{\left|\nabla^{M_{1}^{t} \times M_{2}^{t}} d_{t}\right|_{g(t)}^{2}}{D(t)}-\frac{\left(d_{t}\right)^{2}}{(D(t))^{3}} g_{t}^{i j} \partial_{i} d^{t} \partial_{j} d^{t}
$$

Furthermore, since $\frac{\partial}{\partial t} D(t)=\frac{1}{D(t)}\left(L \frac{\partial L}{\partial t}+d_{t} \frac{\partial d_{t}}{\partial t}\right)$ and using (4.9), the inequality (1.4) can be written

$$
L(t) \frac{\partial L}{\partial t}+d_{t} \frac{\partial d_{t}}{\partial t} \geq d_{t} \Delta_{M_{1}^{t} \times M_{2}^{t}} d_{t}+\left|\nabla^{M_{1}^{t} \times M_{2}^{t}} d_{t}\right|_{g(t)}^{2}-\left(\frac{d_{t}}{D(t)}\right)^{2} g_{t}^{i j} \partial_{i} d_{t} \partial_{j} d_{t}
$$

which simplifies as

$$
L(t) \frac{\partial L}{\partial t}+d_{t} \frac{\partial d_{t}}{\partial t} \geq d_{t} \Delta_{M_{1}^{t} \times M_{2}^{t}} d_{t}+\left(1-\left(\frac{d_{t}}{D(t)}\right)^{2}\right)\left|\nabla^{M_{1}^{t} \times M_{2}^{t}} d_{t}\right|_{g(t)}^{2}
$$

where we used the fact that, for a local basis of tangent vectors $\partial_{i}$ on $M_{1} \times M_{2}$,

$$
\begin{aligned}
\left|\nabla^{M_{1}^{t} \times M_{2}^{t}} d_{t}\right|_{g(t)}^{2} & =g_{t}\left(\nabla^{M_{1}^{t} \times M_{2}^{t}} d_{t}, \nabla^{M_{1}^{t} \times M_{2}^{t}} d_{t}\right) \\
& =\left(g_{t}\right)_{i j} g_{t}^{a i} \partial_{a} d_{t} \cdot g_{t}^{b j} \partial_{b} d_{t} \\
& =g_{t}^{i j} \partial_{i} d_{t} \cdot \partial_{j} d_{t} .
\end{aligned}
$$

To make this construction more explicit, consider

Example 4.1. $M_{1} \cong M_{2} \cong$ the flat torus $\mathbb{T}^{2}$.

Let $\left(M_{1}, g_{1}(t)\right) \cong\left(M_{2}, g_{2}(t)\right) \cong\left(\mathbb{R}^{2} / \mathbb{Z}^{2}, g_{\mathbb{T}^{2}}\right) \cong\left(\mathbb{S}^{1} \times \mathbb{S}^{1},\left(\frac{1}{2 \pi}\right)^{2} d x^{2}+\left(\frac{1}{2 \pi}\right)^{2} d y^{2}\right)$. Since $\operatorname{Ric}_{\mathbb{T}^{2}} \equiv 0$, the flat torus is a stationary point for the Ricci flow and thus, $g_{i}(t) \equiv g_{\mathbb{T}^{2}}$, for all $t$, and $i=1$, 2. Define a family of metrics $D(t): M_{1} \sqcup M_{2} \times M_{1} \sqcup M_{2} \rightarrow \mathbb{R}^{\geq 0}$ by setting

$$
D(t ; \mathbf{a}, \mathbf{b})= \begin{cases}d_{\mathbb{T}^{2}}(\mathbf{a}, \mathbf{b}), & \text { if } \mathbf{a}, \mathbf{b} \in M_{i} \\ \sqrt{L^{2}(t)+d_{\mathbb{T}^{2}}^{2}(\phi(\mathbf{a}), \mathbf{b}),} & \text { if } \mathbf{a} \in M_{1}, \mathbf{b} \in M_{2} \text { or } \mathbf{a} \in M_{2}, \mathbf{b} \in M_{1},\end{cases}
$$

where $\phi: \mathbb{T}^{2} \rightarrow \mathbb{T}^{2}$ is the identity map. To interpret (1.4), we consider $D(t)$ and $d_{t}$ as functions on $\mathbb{T}^{2} \times \mathbb{T}^{2}$ with canonical metric

$$
g_{\mathbb{T}^{2} \times \mathbb{T}^{2}}=g_{\mathbb{T}^{2}} \times g_{\mathbb{T}^{2}}=\left(\frac{1}{2 \pi}\right)^{2}\left[\left(d x^{1}\right)^{2}+\left(d x^{2}\right)^{2}+\left(d x^{3}\right)^{2}+\left(d x^{4}\right)^{2}\right] .
$$

Furthermore, since the metics are stationary, the Laplacian $\Delta_{M_{1}^{t} \times M_{2}^{t}}$ is independent of $t$ and $d_{t}=d$ so that, inside the cut locus,

$$
\Delta_{\mathbb{T}^{2} \times \mathbb{T}^{2}} d(\mathbf{a}, \mathbf{b})=\left.\Delta_{\mathbb{T}^{2}}^{1} d(\cdot, \mathbf{b})\right|_{\mathbf{a}}+\left.\Delta_{\mathbb{T}^{2}}^{2} d(\mathbf{a}, \cdot)\right|_{\mathbf{b}}=\frac{(2 \pi)^{2}}{d_{\mathbf{b}}(\mathbf{a})}+\frac{(2 \pi)^{2}}{d_{\mathbf{a}}(\mathbf{b})}=\frac{2(2 \pi)^{2}}{d(\mathbf{a}, \mathbf{b})}
$$


and thus,

$$
d_{t} \Delta_{M_{1}^{t} \times M_{2}^{t}} d_{t}=d \cdot \Delta_{\mathbb{T}^{2} \times \mathbb{T}^{2}} d=d \cdot \frac{2(2 \pi)^{2}}{d}=2(2 \pi)^{2} .
$$

Also, since $g_{t}^{i j}=g^{i j}$, we have

$$
g_{t}^{i j} \partial_{i} d_{t} \partial_{j} d_{t}=g^{i j} \partial_{i} d \partial_{j} d=(2 \pi)^{2} \sum_{i=1}^{4}\left(\partial_{i} d\right)^{2}=2(2 \pi)^{2}
$$

Lastly, since $\nabla^{\mathbb{T}^{2} \times \mathbb{T}^{2}} d=g^{i j} \partial_{i} d \partial_{j}=(2 \pi)^{2} \delta^{i j} \partial_{i} d \partial_{j}=\sum_{i=1}^{4}(2 \pi)^{2} \partial_{i} d \partial_{i}$, we get

$$
\begin{aligned}
\left|\nabla^{M_{1}^{t} \times M_{2}^{t}} d_{t}\right|_{g(t)}^{2}=\left|\nabla^{\mathbb{T}^{2} \times \mathbb{T}^{2}} d\right|^{2} & =g_{i j}\left(\nabla^{\mathbb{T}^{2} \times \mathbb{T}^{2}} d\right)^{i}\left(\nabla^{\mathbb{T}^{2} \times \mathbb{T}^{2}} d\right)^{j} \\
& =(1 / 2 \pi)^{2} \delta_{i j}\left(\nabla^{\mathbb{T}^{2} \times \mathbb{T}^{2}} d\right)^{i}\left(\nabla^{\mathbb{T}^{2} \times \mathbb{T}^{2}} d\right)^{j} \\
& =\sum_{i=1}^{4}(1 / 2 \pi)^{2}\left[\left(\nabla^{\mathbb{T}^{2} \times \mathbb{T}^{2}} d\right)^{i}\right]^{2} \\
& =\sum_{i=1}^{4}(1 / 2 \pi)^{2}\left[(2 \pi)^{2} \partial_{i} d\right]^{2} \\
& =\sum_{i=1}^{4}(2 \pi)^{2}\left(\partial_{i} d\right)^{2}=\left|\nabla^{1} d\right|^{2}+\left|\nabla^{2} d\right|^{2}=2(2 \pi)^{2} .
\end{aligned}
$$

Therefore, in this setting (4.11) becomes

$$
L(t) \frac{\partial L}{\partial t} \geq 4(2 \pi)^{2}-2(2 \pi)^{2}\left(\frac{d}{D(t)}\right)^{2} .
$$

Roughly estimating $0 \leq d / D(t) \leq 1$, we can take $L(t)=2 \pi \sqrt{8 t+L^{2}(0)}$ so that $L \frac{\partial L}{\partial t}=4(2 \pi)^{2}$ which satisfies (4.25). Naturally, any $L(t)$ with growth larger than $t^{1 / 2}$ would also satisfy condition (1.4) as well and give another family of distance metrics satisfying the super Ricci flow on the disjoint union.

Remark 4.2. In general, for $M_{1} \cong M_{2} \cong\left(\mathbb{R}^{n} / \mathbb{Z}^{n}, g_{\mathbb{T}^{n}}\right)$ we would have $L(t)=2 \pi \sqrt{4 n t+L^{2}(0)}$.

Remark 4.3. A variation of this construction can be used for $M_{1}$ and $M_{2}$ which are only assumed to be homeomorphic. In the definition of $D(t)$ given in (4.2), take, for $\mathbf{a} \in M_{1}, \mathbf{b} \in M_{2}$

$$
\min \left(\inf _{\phi} \sqrt{\left.L^{2}(t)+d_{g_{2}(t)}^{2}(\phi(\mathbf{a}), \mathbf{b})\right)}, \inf _{\phi} \sqrt{L^{2}(t)+d_{g_{1}(t)}^{2}\left(\mathbf{a}, \phi^{-1}(\mathbf{b})\right)}\right),
$$

where the infimum is taken over all homeomorphisms $\phi: M_{1} \rightarrow M_{2}$ and, as before, $L(t)$ depends only on $t$.

Acknowledgement: This research was sponsored in part by the National Science Foundation Grants OISE \#0754379 and DMS \#1006059. We would like to thank The Graduate Center at CUNY where part of this work was completed and Prof. Sormani for her interest and support. In addition, MM would like to thank the Mathematics Institute of the University of Warwick and Peter Topping for their hospitality as this work was completed. SL also thanks Dan Knopf for helpful discussions during his visit to U Texas at Austin.

\section{References}

[1] Angenent, S. and Knopf, D., An example of neck-pinching for Ricci flow on $\mathbb{S}^{n+1}$. Math. Res. Lett. 11 (2004), no. 4, $493-518$.

[2] Angenent, S. and Knopf, D., Precise asymptotics of the Ricci flow neckpinch. Comm. Anal. Geom. 15 (2007), no. 4, $773-844$.

[3] Angenent, S., Caputo, C., and Knopf, D. Minimally invasive surgery for Ricci flow singularities. J. Reine Angew. Math. 672 (2012), 39-87. 
[4] Bakry, D. and Emery, M. Diffusions hypercontractives. In Séminaire de probabilités, XIX, 1983/84, volume 1123 of Lecture Notes in Math., pp 177-206. Springer, Berlin, 1985.

[5] Böhm, C. and Wilking, B. Manifolds with positive curvature operators are space forms. Ann. of Math. (2) 167 (2008), no. 3 , 1079-1097.

[6] Cordero-Erausquin, D., McCann, R., and Schmuckenschläger, M. Prèkopa-Leindler type inequalities on Riemannian manifolds, Jacobi fields, and optimal transport Ann. Fac. Sci. Toulouse Math. (6) 15 (2006), no. 4, 613-635.

[7] Cordero-Erausquin, D., McCann, R., and Schmuckenschläger, M. A Riemannian interpolation inequalityà la Borell, Brascamp and Lieb, Invent. Math. 146 (2001), no. 2, 219-257.

[8] Ethier, S.N. and Kurtz, T. G. Markov processes. Characterization and convergence. Wiley Series in Probability and Mathematical Statistics: Probability and Mathematical Statistics. John Wiley \& Sons, Inc., New York, 1986.

[9] Feldman, M., Ilmanen, T. and Knopf, D., Rotationally symmetric shrinking and expanding gradient Kahler-Ricci solitons. J. Differential Geom. 65 (2003), no. 2, 169-209.

[10] Hamilton, R. Three-manifolds with positive Ricci curvature. J. Differential Geom. 17 (1982), no. 2, 255-306.

[11] Hamilton, R. Four-manifolds with positive curvature operator. J. Differential Geom. 24 (1986), no. 2, 153-179.

[12] Kleiner, B. and Lott, J. Singular Ricci flows I. arXiv:1408.2271v1 [math.DG].

[13] Lott, J. and Villani, C., Ricci curvature for metric-measure spaces via optimal transport. Ann. of Math. (2) 169 (2009), no. 3 , 903-991.

[14] Topping, P. and McCann, R., Ricci flow, entropy and optimal transportation. Amer. J. Math. 132 (2010), no. 3, 711-730.

[15] Perelman, G. The entropy formula for the Ricci flow and its geometric applications, arXiv:math/0211159v1 [math.DG].

[16] Sesum, N., Curvature tensor under the Ricci flow. Amer. J. Math. 127 (2005), no. 6, 1315-1324.

[17] Simon, M. A class of Riemannian manifolds that pinch when evolved by Ricci flow. Manuscripta Math. 101 (2000), no. 1, 89 . 114.

[18] Sturm, K.T. On the geometry of metric measure spaces. I. Acta Math. 196 (2006), no. 1, 65-131.

[19] Sturm, K.T. On the geometry of metric measure spaces. II. Acta Math. 196 (2006), no. 1, 133-177.

[20] Sturm, K.T. and von Renesse, M., Transport inequalities, gradient estimates, entropy, and Ricci curvature. Comm. Pure Appl. Math. 58 (2005), no. 7, 923-940.

[21] Topping, P. Lectures on the Ricci flow. London Mathematical Society Lecture Note Series, 325. Cambridge University Press, Cambridge, 2006.

[22] Vuillermot, P. A generalization of Chernoff's product formula for time-dependent operators. J. Funct. Anal. 259 (2010), no. 11, 2923-2938. 